\title{
Effect of insertion torque on titanium implant osseointegration: an animal experimental study
}

\section{Authors}

- Joke Duyck,

Corresponding author

1. Department of Oral Health Sciences \& Dental Clinic, KU Leuven \& University Hospitals Leuven, BIOMAT KU Leuven \& Prosthetics Unit, Leuven, Belgium

o Corresponding author:

Joke Duyck

Department of Oral Health Sciences \& Dental Clinic

KU Leuven \& University Hospitals Leuven

BIOMAT KU Leuven \& Prosthetics Unit

Kapucijnenvoer 7 blok a box 7001

BE-3000 Leuven

Belgium

Tel.: +3216332468

Fax: +3216332309

e-mail: joke.duyck@uzleuven.be

- Rutger Roesems,

0. Department of Oral Health Sciences \& Dental Clinic, KU Leuven \& University Hospitals Leuven, BIOMAT KU Leuven \& Prosthetics Unit, Leuven, Belgium

- Marcio V. Cardoso,

0. Department of Oral Health Sciences \& Dental Clinic, KU Leuven \& University Hospitals Leuven, BIOMAT KU Leuven \& Prosthetics Unit, Leuven, Belgium

- Toru Ogawa,

0. Division of Advanced Prosthetic Dentistry, Tohoku University Graduate School of Dentistry, Sendai, Japan 


\section{- Germana De Villa Camargos,}

0. Department of Oral Health Sciences \& Dental Clinic, KU Leuven \& University Hospitals Leuven, BIOMAT KU Leuven \& Prosthetics Unit, Leuven, Belgium

1. Department of Prosthodontics and Periodontology, State University of Campinas, Piracicaba Dental School, Piracicaba, SP, Brazil

\section{- Katleen Vandamme}

0. Department of Oral Health Sciences \& Dental Clinic, KU Leuven \& University Hospitals Leuven, BIOMAT KU Leuven \& Prosthetics Unit, Leuven, Belgium

- First published: 11 December 2013

- DOI: $10.1111 /$ clr.12316

\section{Abstract}

\section{Objective}

To evaluate the effect of implant insertion torque on the peri-implant bone healing and implant osseointegration.

\section{Material and methods}

Bilaterally in the tibia of five adult New Zealand white rabbits, 20 implants were installed, subdivided into four groups, corresponding to two insertion torque conditions (low, $<10 \mathrm{Ncm}$ vs. high $>50 \mathrm{Ncm}$ ) and 2 experimental periods (2 weeks vs. 4 weeks of healing). The implant insertion torque was determined by the surgical drill diameter relative to the implant diameter. Implant osseointegration was evaluated by quantitative histology (bone-to-implant contact with host bone [BIC-host], with neoformed bone [BIC-de novo], with both bone types [BICtotal], and peri-implant bone [BA/TA]). Every response was modelled over time using GEE (general estimation equation) with an unstructured variance-covariance matrix to correct for dependency between the measurements from one animal. The statistical significance level of $\alpha=0.05$ was applied.

\section{Results}

Significantly, more BIC-host and BIC-total were recorded for $\mathrm{H}$ implants compared with L implants after 2 week of healing $(P=0.010$ and $P=0.0001$, respectively). However, this result was no longer found for the extended healing period. Furthermore, BIC-total significantly increased over time for $\mathrm{L}$ implants $(P<0.00001)$. In contrast, the significant increase in BA/TA over time was found for $\mathrm{H}$ implants $(P<0.01)$. Finally, $\mathrm{H}$ insertion torque led to an increased BA/TA after 4 week of healing $(P<0.02)$ compared with the $\mathrm{L}$ insertion protocol.

\section{Conclusion}


L insertion torque implants installed in the rabbit tibial bone osseointegrate with considerable de novo bone formation. This bone neoformation enables L implants to catch up, already during the early osseointegration stage, the initial inferior amount BIC contact compared with that of $\mathrm{H}$ implants. A negative impact of the created strain environment accompanying $\mathrm{H}$ insertion torque implant installation on the biological process of osseointegration could not be observed, at least not at tissue level.

The conventional protocol for dental implant rehabilitation recommends an interval of 36 months between surgery and implant loading (Branemark et al. 1985). This procedure enables undisturbed cell interactions with the implant surface, bone healing and implant stabilization. The rationale behind this approach is that implant micromovements caused by functional forces during wound healing may induce fibrous tissue formation rather than bone contact, leading to clinical failure (Szmukler-Moncler et al. 1998). Nevertheless, several experimental studies have shown that immediate or early loading of endosseous titanium implants does not necessarily lead to fibrous tissue healing (Nkenke et al. 2003, 2005; Romanos et al. 2003). In addition, clinical studies have demonstrated high levels of predictability with such immediate implant rehabilitation protocols (Nkenke \& Fenner 2006; Esposito et al. 2013).

In early and immediate loading protocols, primary implant stability is one of the most important factors in achieving predictable treatment outcome (Szmukler-Moncler et al. 2000; Tabassum et al. 2013). This implant-bone interlocking inhibits detrimental micromotion and shear forces at the interface and allows an efficient transfer of potentially stimulating forces to

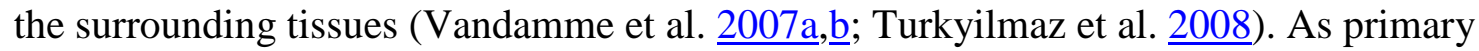
implant stability directly depends on the mechanical connection between the implant and the surrounding bone, it can be strongly influenced by the implant design (Freitas et al. 2012), the bone quality and quantity (Beer et al. 2003) and the surgical technique (drill diameter, depth of the preparation and tapping of the implant site) (Javed \& Romanos 2010; Tabassum et al. 2013). In this context, undersized implant site preparation may increase primary implant stability (Campos et al. 2012). The insertion of the implant after preparing the undersized osteotomy requires a considerable force, which is referred to as the insertion torque. Considering that primary implant stability is influenced by the mechanical interlocking between the implant and the receiving host bone bed, it has been suggested that the implant success can be accelerated and/or enhanced by a surgical protocol applying high insertion torques (Trisi et al. 2009). In the contrast, other studies have suggested that high insertion torque values produce strong compressive forces onto the peri-implant bone, an altered mechanical strain environment and the potential induction of deleterious effects on the local microcirculation and bone cellular responses, which may lead to bone necrosis and ultimately to a retarded or compromised implant osseointegration (Coelho et al. 2010). Thus, while a high primary implant stability is a prerequisite for immediate implant loading, low levels of compressive stresses and strains immediately after implant placement are preferred concomitantly (Freitas et al. 2012).

To the authors' knowledge, little has been published about the quantified implant insertion torque in relation to the in vivo osseointegration process. Therefore, the aim of the present study was to evaluate the role of implant insertion torque on the peri-implant bone healing 
during implant osseointegration. It was hypothesized that implant installation with high insertion torque provides a better primary stability and a higher initial bone-to-implant contact compared with implants installed with low insertion torque, but displaying a delay of the biological process of osseointegration.

\section{Material and methods}

\section{Animals and implant surgery}

Five 4-month-old New Zealand white rabbits were used for this study. The average weight was 3782 g (range: 3350-4110 g). Prior to surgery, the rabbits were pre-anaesthetized with a xylazine (Vexylan ${ }^{\circledR}$, CEVA, Brussels, Belgium) injection intramuscularly at a dose of $1 \mathrm{mg} / \mathrm{kg}$ body weight, combined with an intramuscular injection of ketamine (Ketamine $1000^{\circledR}, \mathrm{CEVA}$ ) at $15 \mathrm{mg} / \mathrm{kg}$ body weight. During surgery, the animals were anaesthetized with propofol (Diprivan ${ }^{\circledR} 1 \%$, AstraZeneca, Brussels, Belgium) at a dose of $0.4 \mathrm{ml} / \mathrm{kg}$ body weight/hour. Postoperatively, the animals were given an intramuscular injection of buprenorfin as analgesics (Temgesic ${ }^{\circledR}$, Schering-Plough, Brussels, Belgium) at $0.05 \mathrm{mg} / \mathrm{kg}$ body weight and penicillin (Penicillin, Kela, Hoogstraten, Belgium) at 300.000 I.U./day. At the end of the experiment, the animals were sacrificed with an overdose of Embutramidemebenzoniumjodide-tetracaïne hydrochloride solution (T61 ${ }^{\circledR}$, Intervet, Mechelen, Belgium, dose: $0.1 \mathrm{ml} / \mathrm{kg}$ bodyweight). The study protocol was approved by the Ethical Committee on Animal Testing of the Katholieke Universiteit Leuven and was performed according to the Belgian animal welfare regulations and guidelines.

In a first surgical intervention, two implants were randomly installed in the tibia of each rabbit, at the mid-diaphyseal region and in a monocortical position. The implants were made of grade 2 commercially pure (c.p.) titanium, with dimensions of $8.0 \times 3.9 \mathrm{~mm}$. The magnitude of insertion torque applied during implant installation was set at either $<10 \mathrm{Ncm}$ ("low") or $>50 \mathrm{Ncm}$ ("high"). The implant insertion torque was determined by the surgical drill diameter relative to the implant diameter and was measured at the time of implant placement by means of the Surgic XT Plus ${ }^{\mathrm{TM}}$ (NSK, Kanuma, Japan) device. For implants installed at low insertion torque $(\mathrm{L})$, the drill diameter $(3.8 \mathrm{~mm})$ was slightly smaller than the outer implant diameter $(3.9 \mathrm{~mm})$, and only the implant screw thread tips were anchored into the host bone. For implants installed at high insertion torque $(\mathrm{H})$, the drill dimension was equal to the inner implant diameter, that is, $3.2 \mathrm{~mm}$. This implied that tapping of the host bed was performed with the implant as such and the screw threads were fully embedded into the host bone. In a second surgical intervention, 2 weeks after the 1st implant insertion, two additional implants were installed in the contralateral tibia, at L vs. $\mathrm{H}$ insertion torque. At week 4, the animals were euthanatized and samples were collected. In total, 20 implants were retrieved that were installed at either $\mathrm{L}(<10 \mathrm{Ncm})$ or $\mathrm{H}(>50 \mathrm{Ncm})$ insertion torque and that healed for either 2 weeks ( $2 \mathrm{wk}$ ) or 4 weeks ( $4 \mathrm{wk}$ ), resulting in five samples per experimental condition.

To get an idea of the effect of low vs. high insertion torque immediately after implant insertion, implants at $\mathrm{L}$ or $\mathrm{H}$ insertion torque were placed in rabbit cadaver tibia ( $n=2$ for both conditions) and histologically observed.

\section{Sample analyses}


The retrieved implants and surrounding bone tissues were isolated and immediately fixed in a $\mathrm{CaCO}_{3}$-buffered formalin solution, dehydrated in an ascending series of ethanol concentration and embedded in methyl methacrylate resin. The tissue blocks containing the implants were cross-sectioned along the direction of the tibia and the implant's long axis by means of a diamond saw (Leica SP1600, Wetzer, Germany) into 95-100- $\mu$ m-thick sections. After polishing to a final thickness of 20-30 $\mu \mathrm{m}$ (Exakt $400 \mathrm{CS}$, Exakt Technologies Inc., Norderstedt, Germany), the sections were stained with a combination of Stevenel's blue and Von Gieson's picrofuchsin red, visualizing mineralized (red) and nonmineralized (blue) tissues. The histological analyses of the sections ( $n=5$ per condition) were performed using a light microscope (Leica Laborlux), which is equipped with a high-sensitivity video camera (AxioCam MRc5, Zeiss, Gottingen, Germany).

The following parameters were measured using an image-analysing software package (Axiovision 4.0, Zeiss) (Fig. 1):
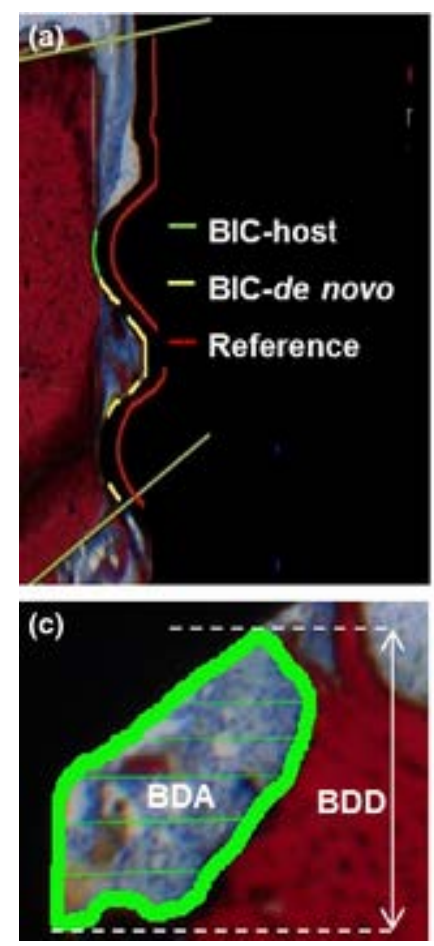

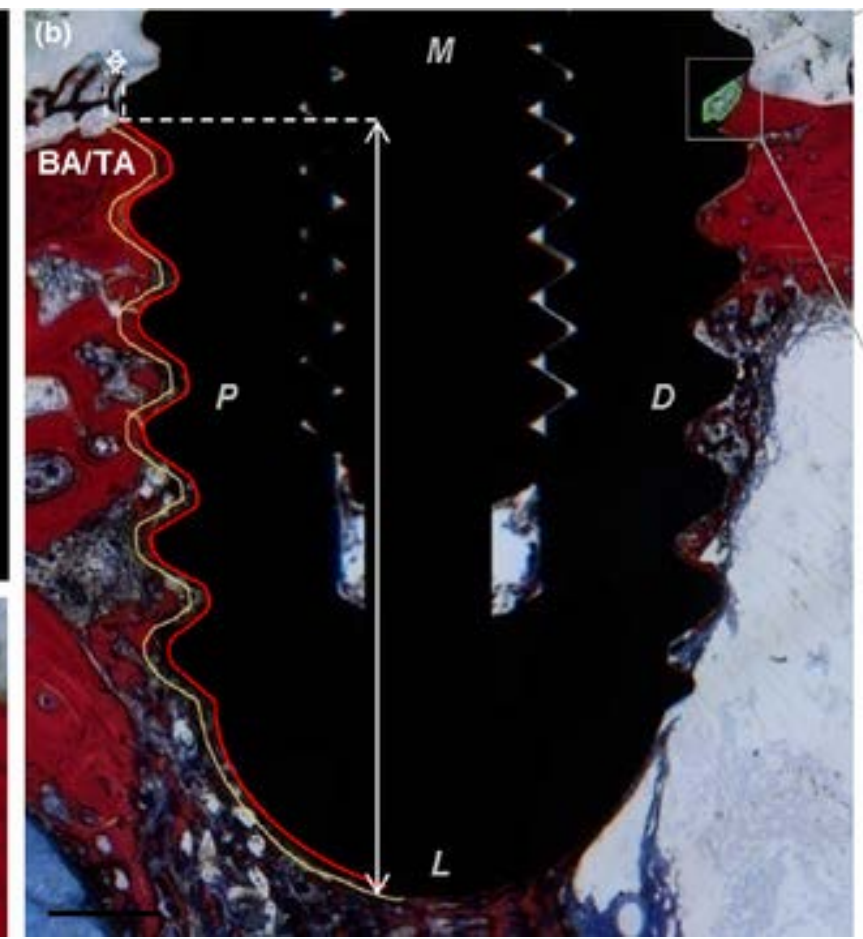

\section{Figure 1.}

Illustrations of the histomorphometrical analyses. (a) contact of the implant with the host bone (BIC-host); contact between the implant and the newly formed bone (BIC-de novo). The reference line corresponds to the width of the cortex; (b) BA/TA, the amount of bone in $100 \mu \mathrm{m}$ area surrounding the implant, over the indicated implant length; (c) bone defect depth $(\mathrm{BDD}, \mu \mathrm{m})$ and bone defect area (BDA, $\mu \mathrm{m} 2)$. Scale bar: $0.5 \mathrm{~mm}$. P, proximal; D, distal; M, medial; L, lateral.

- Implant contact with the host bone (BIC-host; \%): summation of the lengths of contact between the implant and the host bone/implant length corresponding to the width of the cortex;

- Implant in contact with newly formed bone (BIC-de novo; \%): summation of the lengths of contact between the implant and the newly formed bone/implant length corresponding to the width of the cortex; 
- Total bone-to-implant contact (BIC-total; \%): BIC-host + BIC-de novo;

- Peri-implant bone area relative to tissue area (BA/TA; \%): the amount of bone in a specific reference area. The reference area was as the $0-100 \mu \mathrm{m}$ zone extending from the implant surface. The area encompassed the peri-implant tissues in both the cortical and medullar region, at both the proximal and distal side of the implant indeed.

- Bone defect depth (BDD; $\mu \mathrm{m}$ ): the height of a bone defect, extending from the original outer cortical bone level to the deepest point of the bone defect.

- Bone defect area (BDA; $\mu \mathrm{m}^{2}$ ): the surface of the bone defect with the outer cortical bone level as reference.

\section{Statistical analysis}

As the histomorphometrical measurements were clustered into the same animal, GEE (general estimation equation) was used to perform the statistical analysis. The insertion torque ( $\mathrm{L}$ vs. $\mathrm{H}$ ), healing time (2 week vs. 4 week) and the interaction between both were considered as the independent variables for the evaluation of the dependent variables BIC-host, BIC-de novo, BIC-total, BA/TA, BDD and BDA. The variables were modelled over time using an unstructured treatment (i.e. insertion torque) specific mean. That is, the model contained the class variables insertion torque, healing time and their interaction. An unstructured variancecovariance matrix was used to correct the dependency between the measurements from one animal. The significance level was set at $P<0.05$.

\section{Results}

Implant installation in rabbit cadaver tibiae and histological observations confirmed the hypothesized initial situation of the $\mathrm{L}$ and $\mathrm{H}$ implants in minor vs. major contact with the host bone, respectively (Fig. ㄹ).
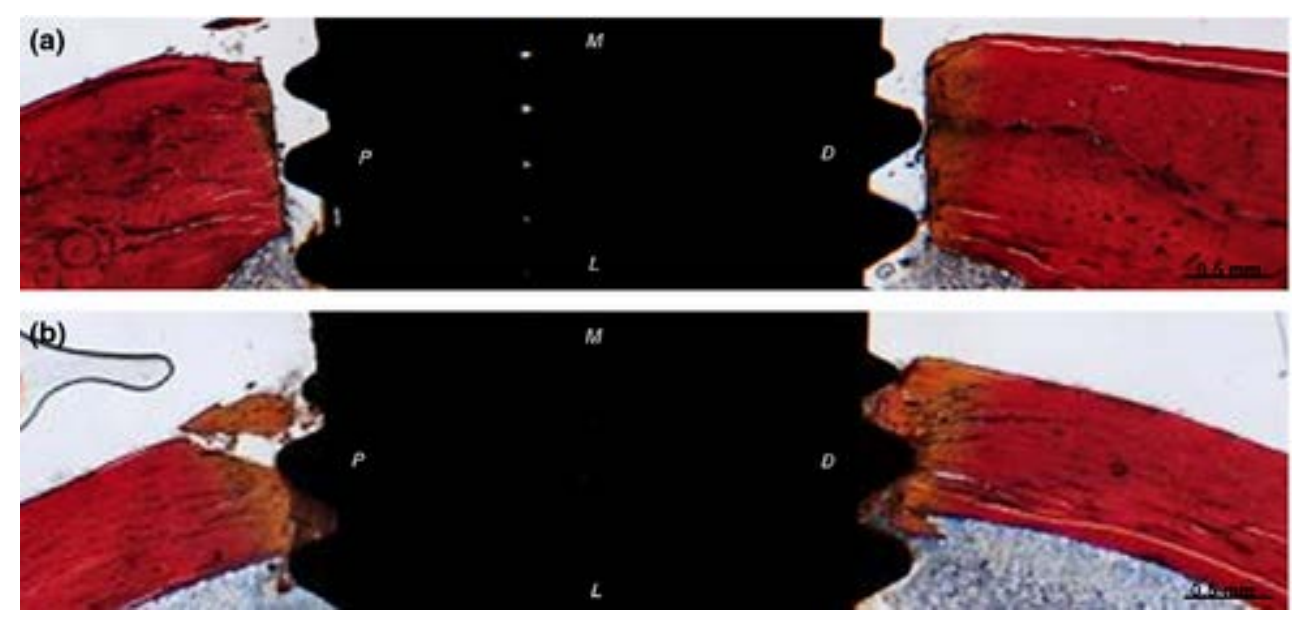

\section{Figure 2.}

Histological observations of implant installation in rabbit cadaver tibiae. (a) L implants in minor contact with the host bone and the presence of a considerable contact-free surface; (b) $\mathrm{H}$ implants in major and direct contact with host bone. Scale bar: $0.5 \mathrm{~mm}$. P, proximal; $\mathrm{D}$, distal; M, medial; L, lateral. 
One implant (of 20) became loose during the course of the study owing to peri-implant infection and was excluded from histomorphometrical analyses. All other implants did not display signs of infection and were clinically stable at the time of animal euthanasia. The histological results revealed little newly formed bone for implants placed with $\mathrm{H}$ insertion torque, but substantial new bone formation for the L implants, along with significant interfacial remodelling for both groups. Hence, to obtain insight into the healing process around these implants, several parameters were quantified in a time-dependent manner.

\section{Bone-to-implant contact}

A distinction was made between the contact of the implant with host (BIC-host) vs. newly formed bone (BIC-de novo), as explained in the Material and Methods section. Four samples could not be evaluated for BIC-host vs. BIC-de novo as the distinction between both was not clear. Statistical GEE analyses revealed significantly higher values for BIC-host for the $\mathrm{H}$ insertion torque group compared with the L group $(P<0.005)$. However, no significant changes in BIC-host within the groups over time were found. Furthermore, no significant interaction effect was found between the independent variables insertion torque and healing time, indicating that there were no significant differences in BIC-host for combinations of insertion torques and healing times. Post hoc tests exploring differences in BIC-host between the different insertion torque regimes revealed that implants installed at $\mathrm{L}$ insertion torque displayed significantly lower BIC-host values compared with implants installed at $\mathrm{H}$ insertion torque after 2 weeks of healing $(10.61 \pm 7.78 \%$ vs. $44.48 \pm 13.72 \% ; P=0.010)$ (Fig. $\underline{3}$ ). This result was no longer found for the 4 -week healing group.

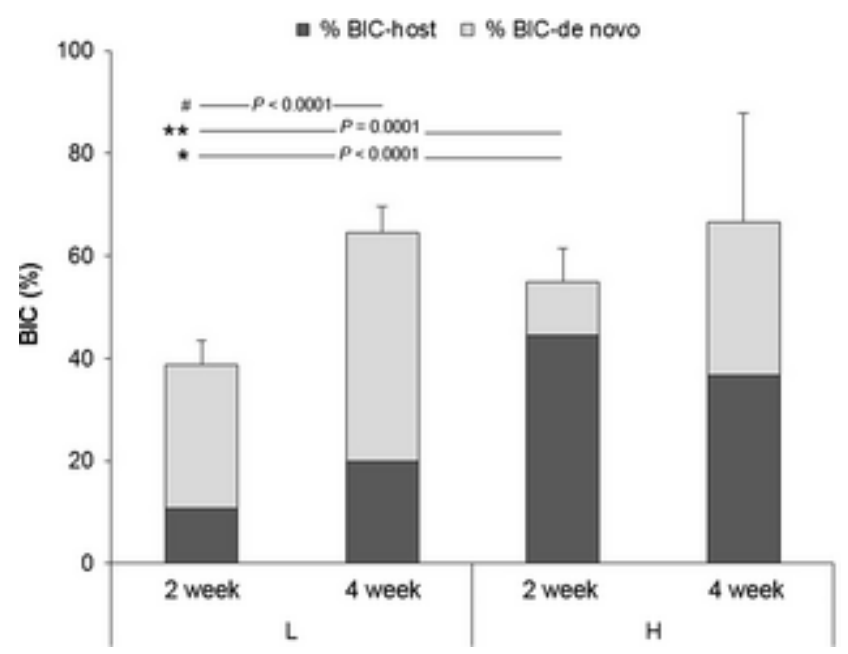

Figure 3.

Histomorphometrical results of the parameter bone-to-implant contact (BIC-host; BIC-de novo; BIC-total). The graph shows mean values and standard deviations of BIC for the different implant insertion torques and healing periods. An asterisk denotes statistically significant differences between the L-H groups for BIC-host. A double asterisk indicates statistically significant differences between the L-H groups for BIC-total. "Indicates differences between the time groups for BIC-total. No significant differences were found for the parameter BIC-de novo. L, low insertion torque; $\mathrm{H}$, high insertion torque.

As BIC-host, significantly different values for the $\mathrm{H}$ insertion torque group compared with the L group were found for BIC-de novo $(P<0.005)$, but without changes over time and without 
interaction effects between the independent variables. Lower BIC-de novo values were obtained when installing the implants at $\mathrm{H}$ insertion torque compared with $\mathrm{L}$ torques ( $10.37 \pm 13.24 \%$ vs. $28.17 \pm 11.94 \%$ for the 2 week condition; $29.84 \pm 13.85 \%$ vs. $44.55 \pm 16.96 \%$ for the 4 week condition). However, these differences were not statistically significant, as shown by the post hoc tests $(P=0.0763$ and $P=0.1024$ for $\mathrm{H}$ and $\mathrm{L}$ comparison at 2 week and 4 week, respectively).

The variable BIC-total was calculated through summation, based on the BIC-host and BIC-de novo values. The obtained values were statistically analysed. BIC-total was significantly influenced by the applied insertion torque $(P<0.05)$. Furthermore, the BIC-total response to specific insertion torque conditions was significantly influenced by the duration of healing, with an increase over time $(P<0.0005)$. No interaction was found between the insertion torque mode and the duration of healing. Post hoc tests revealed a selected, that is, for the 2week healing condition, significant difference for BIC-total in response to the applied insertion torque mode (38.78 $\pm 4.65 \%$ vs. $54.84 \pm 6.58 \% ; P=0.0001$ for $\mathrm{L}$ vs. $\mathrm{H}$ ), as well as a statistical significant increase in BIC-total over time for the L experimental condition (38.78 $\pm 4.65 \%$ vs. $64.45 \pm 5.27 \%$; $P<0.0001$ for 2 week vs. 4 week at $L$ insertion torque) (Fig. $\underline{3}$ ).

\section{Peri-implant bone area relative to tissue area}

The peri-implant bone formation response was significantly influenced by the insertion torque value $(P<0.0001)$ and by the duration of healing $(P<0.0005)$. No interaction was found between these factors. As BIC-host and BIC-total, post hoc analyses revealed selected changes for the peri-implant BA/TA in response to the insertion torque and the healing time. $\mathrm{BA} / \mathrm{TA}$ responses relative to the insertion torque were found to be only significant for the 4week healing time point (36.26 $\pm 7.50 \%$ vs. $26.93 \pm 3.60 \%$ for $\mathrm{H}$ and L, respectively; $P<0.02)$ (Fig. 4). Furthermore, differences over time were recorded only for the H group $(27.45 \pm 7.22 \%$ vs. $36.26 \pm 7.50 \%$ for 2 week and 4 week, respectively; $P<0.01)$ (Fig. 4 ).

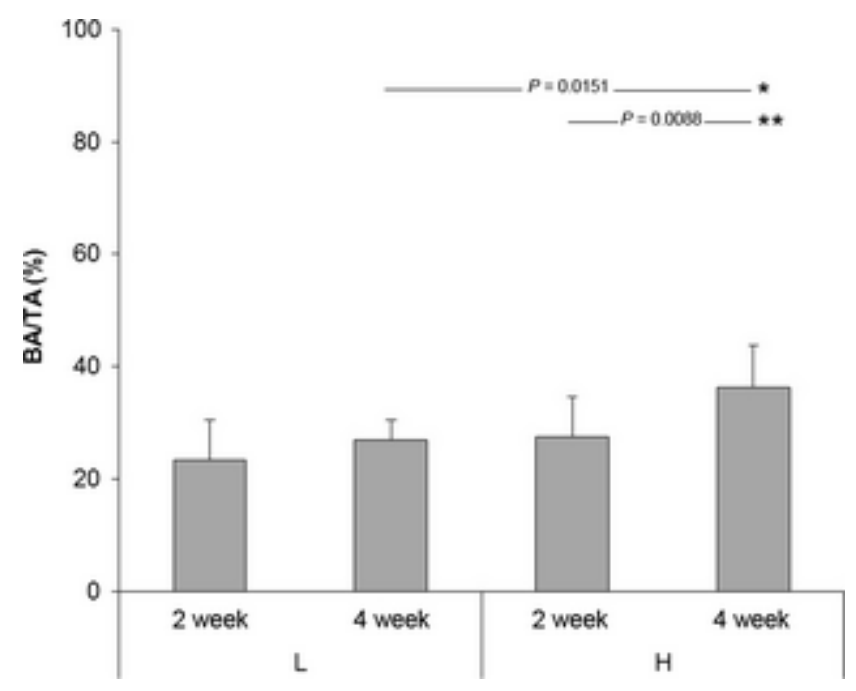

Figure 4. 
Histomorphometrical results of the parameter "peri-implant bone area relative to tissue area" (BA/TA). Mean values and standard deviations of BA/TA for the different implant insertion torques and healing periods are shown. An asterisk denotes statistically significant differences between the L-H groups at 4 weeks of healing. A double asterisk indicates statistically significant differences over time for the $\mathrm{H}$ group (. L, low insertion torque; $\mathrm{H}$, high insertion torque.

\section{Bone defect depth (BDD) and bone defect area (BDA)}

As bone defects were only observed on four of 20 samples, no statistical analysis could be performed. For the observed bone defects (2 originating from H-2 week group, 1 from L2 week group and 1 from H-4 week group), the mean BDD and BDA were $191.2 \mu \mathrm{m}$ (range 95-335 $\mu \mathrm{m}$ ) and $15 \mu \mathrm{m}^{2}$ (range 4.4-26.7 $\mu^{2}$ ), respectively.

\section{Discussion}

Immediate or early implant loading with shortened healing periods when compared to the conventional 3-6 months load-free period of the original Brånemark protocol has been performed with high levels of predictability for some implant systems (Esposito et al. 2013). For such treatment modalities, primary implant stability has been regarded as a key factor for treatment success (Lioubavina-Hack et al. 2006; Javed \& Romanos 2010). The primary stability of implants and its related clinical implication are not dependent only on the implant geometry and host bone density, but also on surgical techniques (Javed \& Romanos 2010;

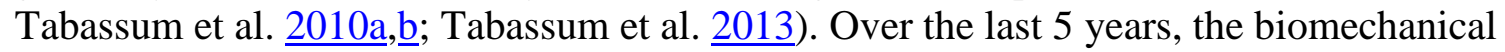
aspects of implant primary stability have been studied by different methodologies such as resonance frequency analysis, histological assessments, insertion or removal torque (Turkyilmaz et al. 2009; Campos et al. 2012; Freitas et al. 2012; Coelho et al. 2013). Despite the ever increasing number of published work in this topic, limited objective (i.e. quantified) information is available on the predictability of different implant surgical protocols and its implication on the implant osseointegration process. Thus, based on the fact that one of the relevant factors that impact primary stability pertains to the implant surgical technique, in particular the implant insertion torque, the present study was undertaken to evaluate the effect of implant insertion torque variation on the implant osseointegration process. To do so, the insertion torque during implant installation was quantified and an extremely low insertion torque was compared with a very high one. It was hypothesized that implant installation with high insertion torque provides a better primary stability compared with implants installed with low insertion torque, but displays a delay of the biological process of osseointegration.

First, to validate the experimental surgical set-up, an ex vivo study was performed prior to the in vivo study. For this, implants belonging to each experimental condition ( $\mathrm{H}$ or $\mathrm{L}$ ) were implanted in cadaver rabbit tibiae. Histological observations of implant installation in rabbit cadaver tibiae at baseline showed that $\mathrm{H}$ implants were to a greater extent in direct contact with host bone compared with L implants, which confirmed the efficacy of placing implants with high insertion torque to improve primary stability. However, quantitative histomorphometry could not be performed in this situation because the cadaver bone at the implant interface broke during implantation of the $\mathrm{H}$ implants group and thereby resulted in bone debris inside the screw threads. This fact can be inter alia attributed to a high bone strain condition that is created when implants are installed with high insertion torque, which may be detrimental for the osseointegration kinetics (Sotto-Maior et al. 2010). Some studies, 
however, report that these bone fragments that are displaced during implant placement, upon their translocation, have osteogenic effects (Dhore et al. 2008; Tabassum et al. 2010a, $\underline{\text { b) }}$.

Similar to the histological observations at baseline, histomorphometrical results at 2 weeks of healing displayed significantly higher BIC-host and BIC-total for the $\mathrm{H}$ group than for the $\mathrm{L}$ group. These observations are the result of the smaller osteotomy that was created for $\mathrm{H}$ implant installation (Campos et al. 2012). In contrast, for the L group, the last drill used had nearly the same diameter as the implant, resulting in a condition where only the tips of the implant were in contact with the surrounding bone and consequently a lower bone-to-implant contact.

While $\mathrm{H}$ implants exhibited high primary mechanical stability, literature indicates that their secondary stability calls for a biological attachment between the foreign body and surrounding bone, which is provided by newly formed bone as well as by remodelled existing bone (Berglundh et al. 2003; Tabassum et al. 2013). Therefore, to evaluate the impact of insertion torque on the different stages of osseointegration (i.e. primary and secondary implant stability), BIC-de novo was measured. Unlike BIC-host and BIC-total at 2 weeks of healing, no significant difference was found for BIC-de novo between the different insertion torque groups neither at 2 nor at 4 weeks, despite the finding of an overall threefold increase in BIC-de novo for L implants. The considerable de novo bone formation in the peri-implant environment is in line with previous studies using an analogous "gap" model and reporting that after 6 weeks of healing, most of the space between implant and host bone has become filled with bone (Berglundh et al. 2003; Slaets et al. 2009). The limited sample number in the present study may be the reason for not having observed significant differences in the de novo formed bone.

For L implants, a trend of more de novo bone formation and a significant increase in BIC-total over time was observed. This can be the consequence of the distinct osseointegration pathways related to different surgical approaches, in which remodelling vs. intramembranouslike ossification are the main pathways for $\mathrm{H}$ and $\mathrm{L}$ implants, respectively (Campos et al. 2012). Previous studies reported that for L implant placement, the void spaces left between the implant and the osteotomy site are filled with a blood clot immediately after implant placement and rapidly replaced by woven bone through an intramembranous-like ossification (Berglundh et al. 2003). In these void spaces, bone can be formed immediately without prior bone resorption. In contrast, for the $\mathrm{H}$ implants, there is pronounced remodelling at the interface region, a condition that may impart the secondary implant stability. This means that the bone in direct contact with the implant, which is responsible for the mechanical primary stability, undergoes resorption before biological integration through bone formation can be established. As a result, the time needed to establish osseointegration might be relatively short for an implant that yields a large contact-free surface compared with an implant with a large contact surface at implant installation (Berglundh et al. 2003). Obviously, the magnitude of the press-fit and the resulting bone necrosis influences the extent of bone remodelling (Berglundh et al. 2003).

Considering the 4-week healing time, a similar BIC-total for $\mathrm{L}$ and for $\mathrm{H}$ implants could be observed, whereas a significant higher BA/TA was found for the $\mathrm{H}$ implants compared with the $\mathrm{L}$ ones. This difference could be attributed to the fact that bone around $\mathrm{H}$ implants originated from the compact host bone, whereas the peri-implant bone around L implants corresponded to newly formed, woven bone. De novo bone at 4 weeks is still considered as immature bone; only after 8-12 weeks of bone healing in the rabbit, immature bone becomes 
mature and more mineralized (Berglundh et al. 2003). Thus, despite the similar BIC-total for $\mathrm{L}$ and $\mathrm{H}$ implants, the lower BA/TA surrounding $\mathrm{L}$ implants may suggest a compromised mechanical condition for the latter implants and consequently prudency when considering loading at this time point.

Future studies assessing clinically relevant insertion torque values for specific implant systems and for different loading protocols, and their impact on the implant outcome are warranted. The reduced sample size can be considered as a limitation in the present study. But another more considerable flaw of the study design is that the experimental conditions that were compared ( $\mathrm{L}$ vs. $\mathrm{H}$ ) were determined at the time of installation by the amount of periimplant bone. The latter parameter at the same time served as an outcome measure and makes the interpretation of the results complicated.

In conclusion, $\mathrm{L}$ insertion torque implants installed in the rabbit tibial bone osseointegrate with considerable de novo bone formation. This bone neoformation without prior bone resorption enables $\mathrm{L}$ implants to catch up, already during the early osseointegration stage, the initial inferior amount of bone-to-implant contact compared with that of $\mathrm{H}$ implants. $\mathrm{H}$ insertion torque implant installation provides not only initially a higher bone-to-implant contact but moreover is anabolic for the bone surrounding the implant. A negative impact of the created strain environment accompanying $\mathrm{H}$ insertion torque implant installation on the biological process of osseointegration could not be observed, at least not at tissue level, thereby rejecting the second part of the study hypothesis. The present results, however, do not give a clear answer of which condition is preferred for clinical application, and factors such as the bone quality, quantity and loading scheme, among others, should be taken into account.

\section{Acknowledgements}

The author would like to acknowledge GC Tech. Europe N.V. (Leuven, Belgium) for providing financial support and Dr. A. Ivanova for the help with the statistical analysis. This work was further supported by the Fund for Scientific Research Flanders (FWO Vlaanderen Postdoctoral researcher K. Vandamme).

\section{References}

- Beer, A., Gahleitner, A., Holm, A., Tschabitscher, M. \& Homolka, P. (2003) Correlation of insertion torques with bone mineral density from dental quantitative CT in the mandible. Clinical Oral Implants Research 14: 616-620.

- Berglundh, T., Abrahamsson, I., Lang, N.P. \& Lindhe, J. (2003) De novo alveolar bone formation adjacent to endosseous implants. Clinical oral implants research 14: 251-262.

- Branemark, P.I., Zarb, G. \& Albrektsson, T. (1985) Tissue-integrated Prostheses Osseointegration in Clinical Dentistry. Chicago: Quintessence.

- Campos, F.E., Gomes, J.B., Marin, C., Teixeira, H.S., Suzuki, M., Witek, L., ZanettaBarbosa, D. \& Coelho, P.G. (2012) Effect of drilling dimension on implant placement torque and early osseointegration stages: an experimental study in dogs. Journal of Oral and Maxillofacial Surgery Official Journal of the American Association of Oral and Maxillofacial Surgeons 70: 43-50. 
- Coelho, P.G., Marin, C., Teixeira, H.S., Campos, F.E., Gomes, J.B., Guastaldi, F., Anchieta, R.B., Silveir, L. \& Bonfant, E.A. (2013) Biomechanical evaluation of undersized drilling on implant biomechanical stability at early implantation times. Journal of Oral and Maxillofacial Surgery: Official Journal of the American Association of Oral and Maxillofacial Surgeons 71: 69-75.

- Coelho, P.G., Suzuki, M., Guimaraes, M.V.M., Marin, C., Granato, R., Gil, J.N. \& Miller, R.J. (2010) Early bone healing around different implant bulk designs and surgical techniques: a study in dogs. Clinical Implant Dentistry and Related Research 12: 202-208.

- Dhore, C.R., Snel, S.J., Jacques, S.V.N., Naert, I.E., Walboomers, X.F. \& Jansen, J.A. (2008) In vitro osteogenic potential of bone debris resulting from placement of titanium screw-type implants. Clinical Oral Implants Research 19: 606-611.

- Esposito, M., Grusovin, M.G., Maghaireh, H. \& Worthington, H.V. (2013) Interventions for replacing missing teeth: different times for loading dental implants. Cochrane Database of Systematic Reviews (Online) 28: CD003878.

- Freitas, A.C. Jr, Bonfante, E.A., Giro, G., Janal, M.N. \& Coelho, P.G. (2012) The effect of implant design on insertion torque and immediate micromotion. Clinical Oral Implants Research 23: 113-118.

- Javed, F. \& Romanos, G.E. (2010) The role of primary stability for successful immediate loading of dental implants. literature review. Journal of Dentistry 38: 612620.

- Lioubavina-Hack, N., Lang, N.P. \& Karring, T. (2006) Significance of primary stability for osseointegration of dental implants. Clinical Oral Implants Research 17: 244-250.

- Nkenke, E. \& Fenner, M. (2006) Indications for immediate loading of implants and implant success. Clinical Oral Implants Research 17: 19-34.

- Nkenke, E., Lehner, B., Fenner, M., Roman, F.S., Thams, U., Neukam, F.W. \& Radespiel-Troger, M. (2005) Immediate versus delayed loading of dental implants in the maxillae of minipigs: follow-up of implant stability and implant failures. The International Journal of Oral Maxillofacial Implants 20: 39-47.

- Nkenke, E., Lehner, B., Weinzierl, K., Thams, U., Neugebauer, J., Steveling, H., Radespiel-Troger, M. \& Neukam, F.W. (2003) Bone contact, growth, and density around immediately loaded implants in the mandible of mini pigs. Clinical Oral Implants Research 14: 312-321.

- Romanos, G.E., Toh, C.G., Siar, C.H., Wicht, H., Yacoob, H. \& Nentwig, G.H. (2003) Bone-implant interface around titanium implants under different loading conditions: a histomorphometrical analysis in the Macaca fascicularis monkey. The Journal of Periodontology 74: 1483-1490.

- Slaets, E., Naert, I., Carmeliet, G. \& Duyck, J. (2009) Early cortical bone healing around loaded titanium implants: a histological study in the rabbit. Clinical oral implants research 20: 126-134.

- Sotto-Maior, B.S., Rocha, E.P., De Almeida, E.O., Freitas-Júnior, A.C., Anchieta, R.B. \& Del Bel Cury, A.A. (2010) Influence of high insertion torque on implant placement: an anisotropic bone stress analysis. Brazilian Dental Journal 21: 508-514.

- Szmukler-Moncler, S., Piattelli, A., Favero, G.A. \& Dubruille, J.H. (2000) Considerations preliminary to the application of early and immediate loading protocols in dental implantology. Clinical Oral Implants Research 11: 12-25.

- Szmukler-Moncler, S., Salama, H., Reingewirtz, Y. \& Dubruille, J.H. (1998) Timing of loading and effect of micromotion on bone-dental implant interface: review of experimental literature. Journal of Biomedical Materials Research 43: 192-203. 
- Tabassum, A., Meijer, G.J., Walboomers, X.F. \& Jansen, J.A. (2013) Evaluation of primary and secondary stability of titanium implants using different surgical techniques. Clinical oral implants research 1-6.

- Tabassum, A., Meijer, G.J., Wolke, J.G.C. \& Jansen, J.A. (2010b) Influence of surgical technique and surface roughness on the primary stability of an implant in artificial bone with different cortical thickness: a laboratory study. Clinical Oral Implants Research 21: 213-220.

- Tabassum, A., Walboomers, X.F., Wolke, J.G.C., Meijer, G.J. \& Jansen, J.A. (2010a) Bone particles and the undersized surgical technique. Journal of Dental Research 89: 581-586.

- $\quad$ Trisi, P., Perfetti, G., Baldoni, E., Berardi, D., Colagiovanni, M. \& Scogna, G. (2009) Implant micromotion is related to peak insertion torque and bone density. Clinical oral implants research 20: 467-471.

- Turkyilmaz, I., Aksoy, U. \& McGlumphy, E.A. (2008) Two alternative surgical techniques for enhancing primary implant stability in the posterior maxilla: a clinical study including bone density, insertion torque, and resonance frequency analysis data. Clinical Implant Dentistry and Related Research 10: 231-237.

- Turkyilmaz, I., Sennerby, L., McGlumphy, E.A. \& Tözüm, T.F. (2009) Biomechanical aspects of primary implant stability: a human cadaver study. Clinical Implant Dentistry and Related Research 11: 113-119.

- Vandamme, K., Naert, I., Geris, L., Vander Sloten, J., Puers, R. \& Duyck, J. (2007a) Influence of controlled immediate loading and implant design on peri-implant bone formation. Journal of Clinical Periodontology 34: 172-181.

- Vandamme, K., Naert, I., Geris, L., Vander Sloten, J., Puers, R. \& Duyck, J. (2007b) The effect of micro-motion on the tissue response around immediately loaded roughened titanium implants in the rabbit. European Journal of Oral Sciences 115: 21-29. 